\title{
A Basis Set for Characterizing Transient Random Phenomena
}

\author{
Roy M. Howard \\ Department of Electrical and Computer Engineering, \\ Curtin University of Technology, GPO Box U 1987, Perth, 6845, Australia.
}

\begin{abstract}
An orthogonal basis consistent with transient random phenomena is proposed and applied to market data and $1 / f$ noise. For $1 / f$ noise the power-rate spectrum is flat. The basis set leads to a power-rate spectrum and can be used to facilitate the detection of specific signal forms.
\end{abstract}

Keywords: Transient random phenomena, basis set, power-rate spectrum, power spectral density. PACS: 02.50.Fz, 05.10.Gg, 05.40.-a

\section{INTRODUCTION}

The scientific endeavour related to characterizing random phenomena has a long and important history, e.g. [1]. One important, and widely used, method for characterizing random phenomena is via waveform decomposition based on utilizing a basis set for a specified interval of interest, e.g. [2]. Usually, the power, or energy, of the constituent waveforms arising from the decomposition are calculated and utilized to define a power spectral density or a time-scale representation for the random process. Common basis sets include the sinusoidal basis set, which leads to the standard, and widely used, power spectral density function, a basis set consistent with a Karhunen-Loève decomposition e.g. [3] and [4], a wavelet basis set, e.g. [5], which leads to a time-scale characterization, etc. In general, the basis sets used are such that the waveforms, or sets of waveforms as in wavelet analysis, exhibit the same form over the interval of interest. As such they are not optimally suited to the characterization of transient random phenomena. For transient random phenomena of a specific type, a custom approach, where the signals in the basis set match the form of the underlying random phenomena, is likely to lead to a better characterization and, hence, a simpler model of the random phenomena.

There have been several papers on orthogonal basis sets for the transient case and typically for the interval [0, ], e.g. [6], [7] and [8]. Such basis sets usually consist of Laguerre, or similar functions, which exhibit exponential decay as infinity is approached. Such basis sets can, naturally, represent transient phenomena of all types. However, in general, the decomposition is likely to be non-optimal when the random phenomenon yields signals which are inconsistent with the basis set signals. In this paper a basis set for characterizing transient phenomena, and consistent with random walk type phenomena is proposed. 


\section{BASIS SET FOR TRANSIENT RANDOM PHENOMENA}

Consider the orthogonal basis set, $\left\{b_{i j}: \boldsymbol{R} \rightarrow \boldsymbol{R}\right\}$, for the interval $\left[0,2^{N} \Delta t\right]$, illustrated in Figure 1 and a signal $x: \boldsymbol{R} \rightarrow \boldsymbol{R}$. The associated data vector $X=$ $\left\{x_{0}, x_{1}, \ldots, x_{2^{N}-1}\right\}$ can be written, using the basis set, as

$$
x_{k}=x(k \Delta t)=\sum_{i=0}^{N} c_{i 0} b_{i 0}(k \Delta t)+\sum_{i=1}^{N-1} \sum_{j=1}^{2^{N-i}-1} c_{i j} b_{i j}(k \Delta t)=\mu_{k}+\sum_{i=1}^{N-1} r_{i}(k \Delta t)
$$

for $k \in\left\{0,1, \ldots, 2^{N}-1\right\}$, where $c_{i j}$ is the coefficient associated with $b_{i j}(t)$, and

$$
\mu_{k}=\sum_{i=0}^{N} c_{i 0} b_{i 0}(k \Delta t), \quad r_{i}(k \Delta t)=\sum_{j=1}^{2^{N-i}-1} c_{i j} b_{i j}(k \Delta t), \quad i \in\{1, \ldots, N-1\}
$$

Accordingly, the data vector can be written as

$$
X=\mu+\sum_{i=1}^{N-1} R_{i}, \quad \mu=\left\{\mu_{0}, \mu_{1}, \ldots, \mu_{2^{N}-1}\right\}, \quad R_{i}=\left\{r_{i}(0), \ldots, r_{i}\left[\left(2^{N}-1\right) \Delta t\right]\right\}
$$

The first component, $\mu$, represents a 'dynamic' mean component consistent with the basis signals $b_{00}(t), b_{10}(t), \ldots, b_{N 0}(t)$. The vector $R_{i}$ is defined by samples of a

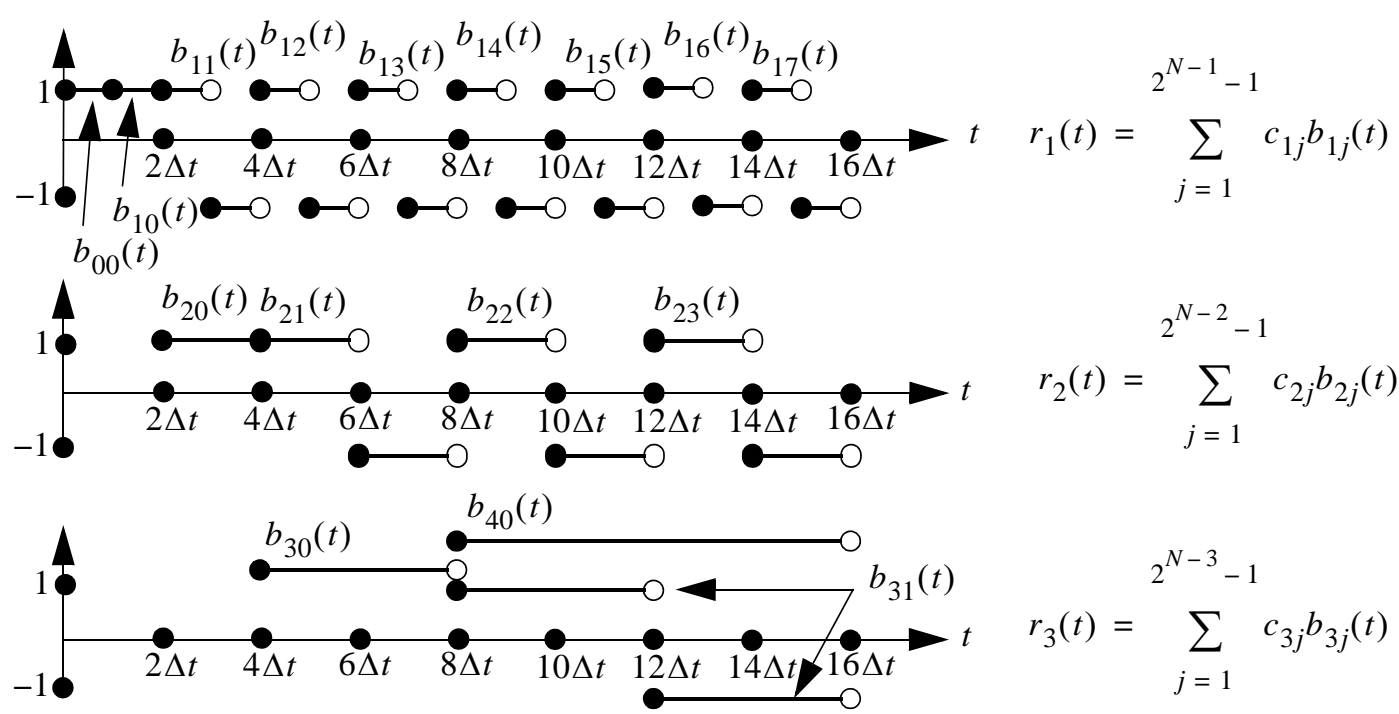

FIGURE 1. Basis set $\left\{b_{00}(t), b_{i j}(t)\right\}, i \in\{1, \ldots, N\}, j \in\left\{0, \ldots, 2^{N-i}-1\right\}$ for $\left[0,2^{N} \Delta t\right)$ and for the case of $N=4$. All waveforms have unity amplitude but some are offset for clarity of display. The basis signals $b_{00}(t), b_{10}(t), \ldots, b_{N 0}(t)$ have a non-zero mean; all other basis signals have a zero mean. The signals $r_{1}, r_{2}$ and $r_{3}$ define the first three signalling random processes. 
signalling random process $r_{i}(t)$, [9], which is based on the basis functions $b_{i 1}(t), \ldots, b_{i, 2^{N-i}-1}(t)$ and has a rate of $\lambda_{i}=1 / 2^{i} \Delta t$. Its first $2^{i}$ components are zero consistent with transient phenomena. Given a data vector $X$ it can be readily shown that the coefficients in the basis decomposition are given by

$$
\begin{gathered}
c_{00}=x_{0}, \quad c_{i 0}=\frac{1}{2^{i-1}} \sum_{k=0}^{2^{i-1}-1} x_{2^{i-1}+k}, \quad i \in\{1,2, \ldots, N\} \\
c_{i j}=\frac{1}{2^{i}}\left[\sum_{k=0}^{2^{i-1}-1} x_{2^{i} j+k}-\sum_{k=0}^{2^{i-1}-1} x_{2^{i} j+2^{i-1}+k}\right], \quad\left\{\begin{array}{l}
i \in\{1, \ldots, N-1\} \\
j \in\left\{1, \ldots, 2^{N-i}-1\right\}
\end{array}\right.
\end{gathered}
$$

The average power, $P_{i}$, in the ith signalling random process $r_{i}(t)$, over the interval $\left[2^{i} \Delta t, 2^{N} \Delta t\right]$, where it is non-zero, is

$$
P_{i}=\frac{1}{\left(2^{N}-2^{i}\right) \Delta t} \int_{2^{i} \Delta t}^{2^{N} \Delta t} r_{i}^{2}(t) d t=\frac{1}{2^{N-i}-1} \sum_{j=1}^{2^{N-i}-1} \boldsymbol{E}\left[c_{i j}^{2}\right]
$$

where $\boldsymbol{E}$ is the expectation operator. For the case of $\boldsymbol{E}\left[c_{i j}^{2}\right]=\boldsymbol{E}\left[c_{i k}^{2}\right]=\sigma_{i}^{2}$, for $k \neq j$, it follows that $P_{i}=\sigma_{i}^{2}$. For a random walk with a step size of $\pm \Delta$, and a step variance of $\sigma^{2}=\Delta^{2}$, it can be shown, utilizing the independence between step increments, that the average power in the ith signalling random process $R_{i}$ is:

$$
P_{i}=\frac{\sigma^{2}}{4}+\frac{2^{i} \sigma^{2}}{12} \cdot\left(1-\frac{1}{2^{i-1}}\right)\left(1-\frac{1}{2^{i}}\right)=\frac{\sigma^{2}}{4}+\frac{\Delta t \sigma^{2}}{12 \lambda_{i}} \cdot\left(1-\frac{1}{2^{i-1}}\right)\left(1-\frac{1}{2}\right)
$$

for $i \in\{1, \ldots, N-1\}$. The second form arises as $\lambda_{i}=1 / 2^{i} \Delta t$ and, thus, the power-rate spectrum exhibits a 1 /rate form.

\section{RESULTS AND DISCUSSION}

Results taken from Forex data (AUD vs USD) and $1 / f$ noise are shown in Figure 2 and clearly show, for the Forex data, 'reasonable market' random walk behaviour at a macro level. Note that the power-rate spectrum for $1 / f$ noise exhibits, consistent with the model proposed in [10], a flat power-rate spectrum. Thus, relative to the proposed 
basis set $1 / f$ noise appears as 'white' noise. The following is a summary of power-rate spectral forms: White noise (flat power spectral density) yields a power-rate spectrum varying according to rate, $1 / f$ noise yields a flat power-rate spectrum and a $1 / f^{2}$ (a power spectral density consistent with a random walk - see ch. 6 of [9]) yields a 1 /rate power-rate spectral form. The proposed basis set can be used to facilitate a test of whether a random phenomena is consistent with a random walk, or has a component which is consistent with a random walk or $1 / f$ noise. Finally, the proposed basis set can yield greater sensitivity to certain types of random phenomena than a standard power spectral density.

power-rate spectrum
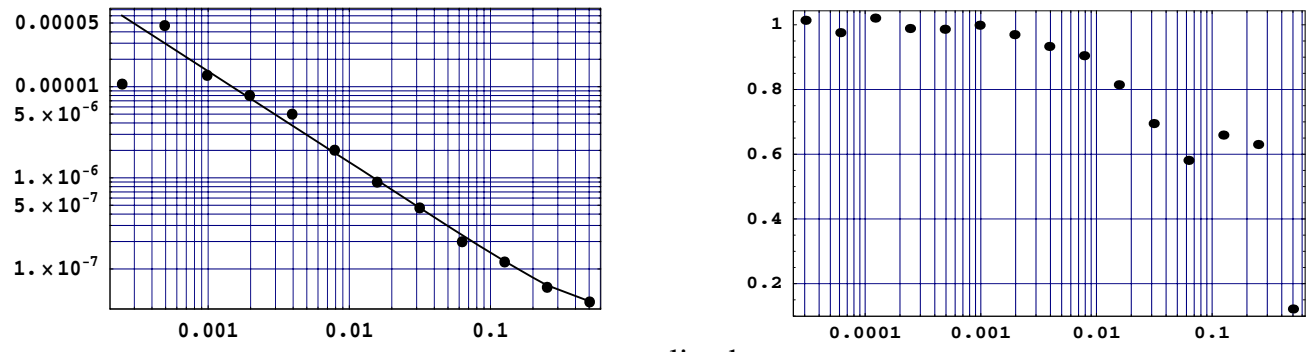

normalized rate

FIGURE 2. Left: Theoretical power-rate spectrum for a random walk (continuous curve) and power-rate spectrum of 4096 values of AUD:USD Forex data (dots) taken with a 10 minute step interval. Right: averaged power-rate spectrum from $1 / f$ noise simulated consistent with [10] and then filtered. 100 averages and 65536 samples have been used. The roll-off is due to the filtering.

\section{CONCLUSION}

An orthogonal basis consistent with transient random phenomena has been proposed and applied to market data and $1 / f$ noise. The basis set leads to a power-rate spectrum and for a random walk the associated power-rate spectrum exhibits a 1 /rate form whilst for $1 / f$ noise the associated power-rate spectrum is constant. The proposed basis set can facilitate the detection of specific signal forms.

\section{REFERENCES}

1. L. Cohen, IEEE Signal Processing Magazine, 20-45, Nov. (2005).

2. L. Debnath and P. Mikusinski, Introduction to Hilbert Spaces with Applications, Academic Press, 1999.

3. M. Loeve, Probability Theory: Part II, Springer Verlag, 4th ed. 1978, p. 144.

4. J. Ardnt, H. Hertzel, S. Bose, M. Falcke and E. Schöll, E. (1997), Chaos, Solitons \& Fractals, 8, 1911-1920 (1997).

5. T. K. Sarkar, C. Su, R. Adve, M. Salazar-Palma, L. Garcia-Castillo and R. Boix, IEEE Antennas and Propagation Magazine, 40, 49-70 Oct., 36-49 Dec. (1998).

6. H. L. Armstrong, IRE Transactions on Circuit Theory, 4, 286-287 (1957).

7. H. L. Armstrong, IRE Transactions on Circuit Theory, 7, 351-354 (1959).

8. J. W. Head, Proceedings of the Cambridge Philosophical Society, 52, 640-651 (1956).

9. R. M. Howard, Principles of Random Signal Analysis and Low Noise Design: The Power Spectral Density and its Applications, Wiley, 2002, ch. 5.

10. R. M. Howard and L. A. Raffel, 'General models for 1/f noise' in Noise in Devices and Circuits, edited by M. J. Deen et. al, SPIE vol. 5113, SPIE, Bellingham, WA, 2003, pp. 282-293. 